doi:10.4149/neo_2017_301

\title{
Detection and characterization of ciRS-7: a potential promoter of the development of cancer
}

\section{Minireview}

\author{
X. B. ZHENG ${ }^{*}$, M. ZHANG*, M. Q. XU*
}

Department of Liver and Vascular Surgery Liver Transplantation Center, West China Hospital, Sichuan University, Chengdu 610041, Sichuan Province, China

${ }^{*}$ Correspondence: xumingqing0018@163.com

${ }^{*}$ Contributed equally to this work.

\section{Received September 8, 2016 / Accepted November 11, 2016}

\begin{abstract}
Circular RNAs (circRNAs) are a class of newly-identified non-coding RNA molecules. CircRNAs are conserved across different species and display specific organization, sequence, and expression in disease. Moreover, circRNAs' closed ring structure, insensitivity to RNase, and stability are advantages over linear RNAs in terms of development and application as a new kind of clinical marker. In addition, according to recent studies, circular RNA-7 (ciRS-7) acts as a sponge of miR-7 and thus inhibits its activity. Numerous evidences have confirmed expression of miR-7 is dysregulated in cancer tissues, however, whether ciRS-7 invovled in oncogenesis by acting as sponge of miR-7 remains unclear. Most recently, a study reported ciRS-7 acted as an oncogene in hepatocellular carcinoma through targeting miR-7 expression. This suggest ciRS-7/ miR-7 axis affects oncogenesis, and it provides a new perspective on the mechanisms of decreased miR-7 expression in cancer tissues. Discovery of sponge role of circRNAs caused researchers to more closely explore the underlying mechanism of carcinogenesis and has significant clinical implications, and may open a new chapter in research on the pathology and treatment of cancers. This review summarizes the structure and function of circRNAs and provides evidence for the impact of ciRS-7 in promoting the development of cancer by acting as sponge of miR-7.
\end{abstract}

Key words: circRNAs, promoters, cancer, $m i R N A$

Circular RNAs (circRNAs) are a class of newly-identified non-coding RNA (ncRNA) molecules, and involved in posttranscriptional regulation like miRNA, long non-coding RNA (lncRNA) and other regulatory RNAs. In the past, the central dogma asserted that RNA's primary role was that of messenger RNA (mRNA), but with the development of a purification method for circRNA molecules combined with improved high-throughput sequencing technology and data analysis methods, thousands of circRNA molecules have been found in humans, leading to new understanding of RNA's many functions [1]. Because they form a closed continuous loop, circRNAs are more stable, and less sensitive to RNase R than linear RNAs, both advantages in the development and application of new diagnostic markers. Although the specific biological functions of circRNA are poorly understood, the two Nature studies indicate that some circRNA compete with endogenous RNA (ceRNA) in gene expression regulation [2, 3]. They also directly bind to target miRNAs to inhibit their expression levels. In addition, circRNA may be an important class of posttranscriptional regulatory factors that can regulate levels of other RNAs and protein activity and act as templates for protein synthesis (Fig 1).

Antisense to the cerebellar degeneration-related protein 1 transcript (CDR1as, also known as the ciRS-7) is a recentlydiscovered circular RNA associated with human disease. This circRNA has at least 70 conservative binding sites for miRNA, so it acts as a miRNA sponge to regulate the expression of miRNA target genes $[2,3]$. Studies have found that CDR1as, together with miR-7, is highly expressed in developing midbrain regions. In zebrafish embryos, overexpression of ciRS-7 


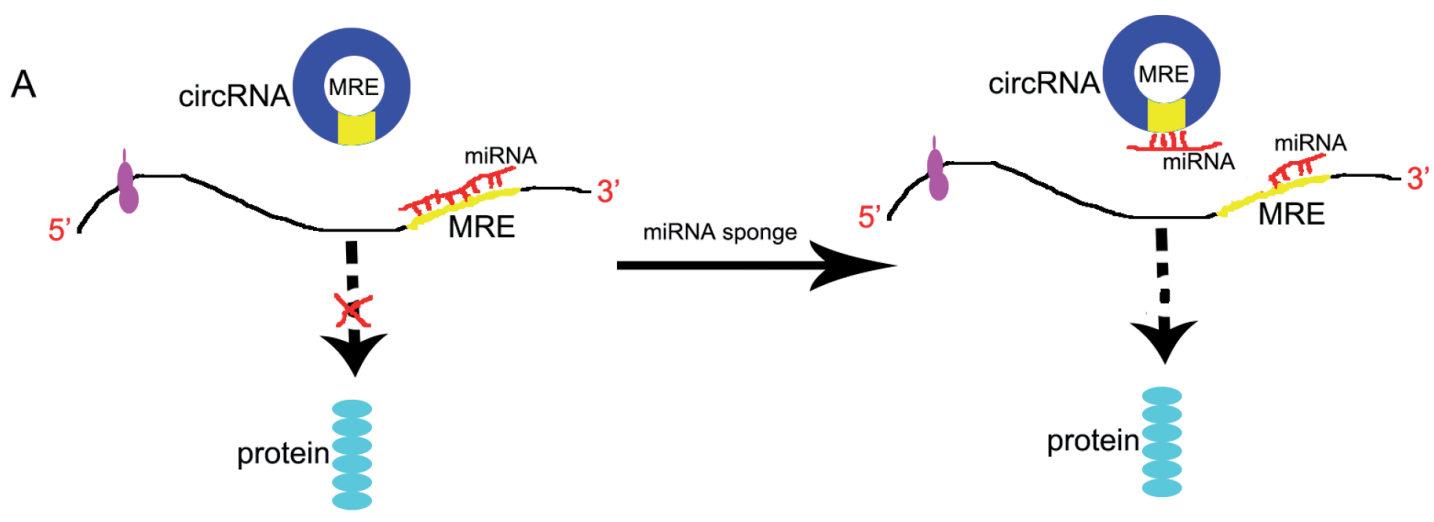

B

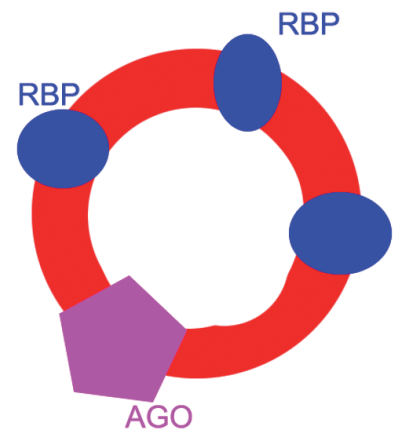

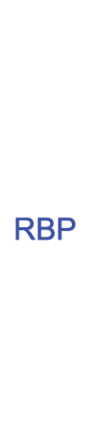

C

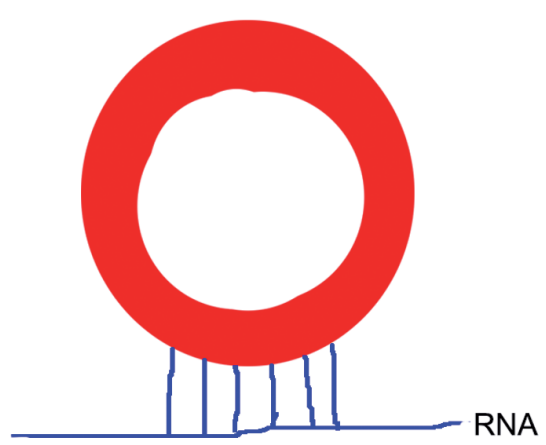

Figure 1. Biological functions of circRNA. (A). CircRNA functions as a miRNA sponge to promote the expression of some of miRNA's targets. (B). CircRNA combines with RNA binding protein (RBP) and AGO protein. (C). CircRNA directly binds base of other RNAs.

reduces the volume of the midbrain, but the damage can be prevented by supplying the miR-7 precursor. This suggests that ciRS-7 may exert a biological effect by interacting with miR-7. The roles of ciRS-7 in the process of cancer initiation and progression has also gathered prominence. Most recently, a study reported ciRS-7 acted as an oncogene in hepatocellular carcinoma through targeting miR-7 expression[4]. Cancer remains as one of the leading causes of mortality worldwide [5], therefore it remains imperative to clarify the underlying mechanisms and novel therapies to improve the survival rate of cancer patients. Deciphering ciRS-7 interplay with miR-7 in cancer would likely confer ciRS-7 great potential to become new diagnostic markers in early stages of cancer, and raise possibility for novel therapeutic interventions.

In this review, we provide a concise and up to date overview of circRNAs, and in particular discuss the context in which ciRS-7 play a role of cancer development by acting as sponge of miR-7.

\section{Mechanism of circRNA formation}

Generally speaking, a normal RNA splicing reaction has two transesterifications: (1) An adenine nucleotide in the branch site attacks a 5' splicing site upstream through 2'-hydroxy, dissociating upstream exons from introns and making lariat introns and exon intermediates downstream; and (2) The 3'-hydroxy located on the end of the exon splicing site upstream attacks a splicing site downstream and connects upstream and downstream exons sequentially to form mature, linear RNA molecules, which can release the remaining lariat RNA fragments [6]. However, in eukaryotes there is a special back splicing which links the upstream sequence of exons reversely with the downstream sequence of exons. This process is called exon cyclization through reverse splicing, or "exon circularization," and the final product is circRNA. Although circRNA formed by exon cyclization was first found in mammalian cells decades ago [7-9], levels of this type of circRNA are very low, and it was considered to be a byproducts of erroneous splicing and long ignored [10].

Brown et al. [11] reports that hundreds of human gene transcriptions are initiated by a classical process where RNA molecules are spliced into rings. These circRNAs consist of a large chunk of identified transcript, indicating that the circRNAs are more abundant and important than previously thought. The study used new analytical methods to show that the circRNAs may be part of a biological process which has not yet been found. 
A

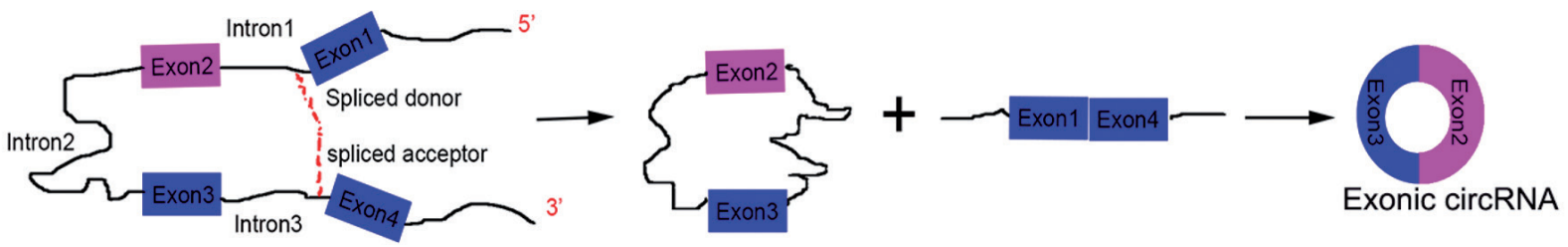

B
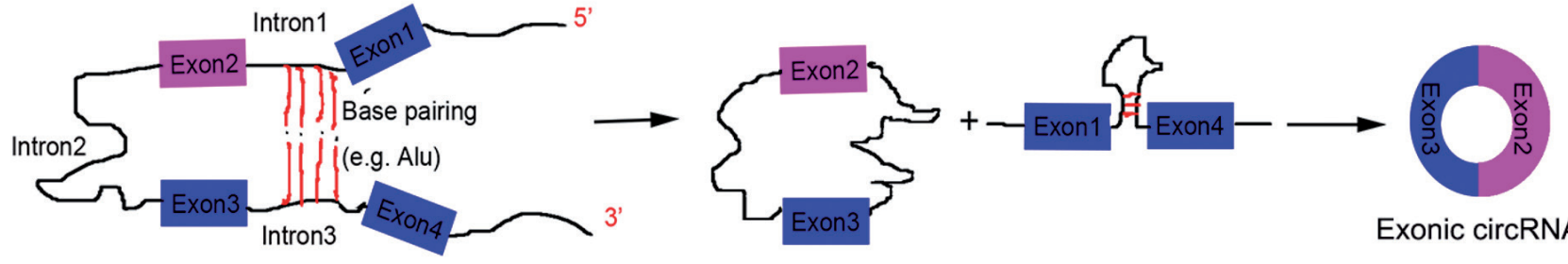

Exonic circRNA
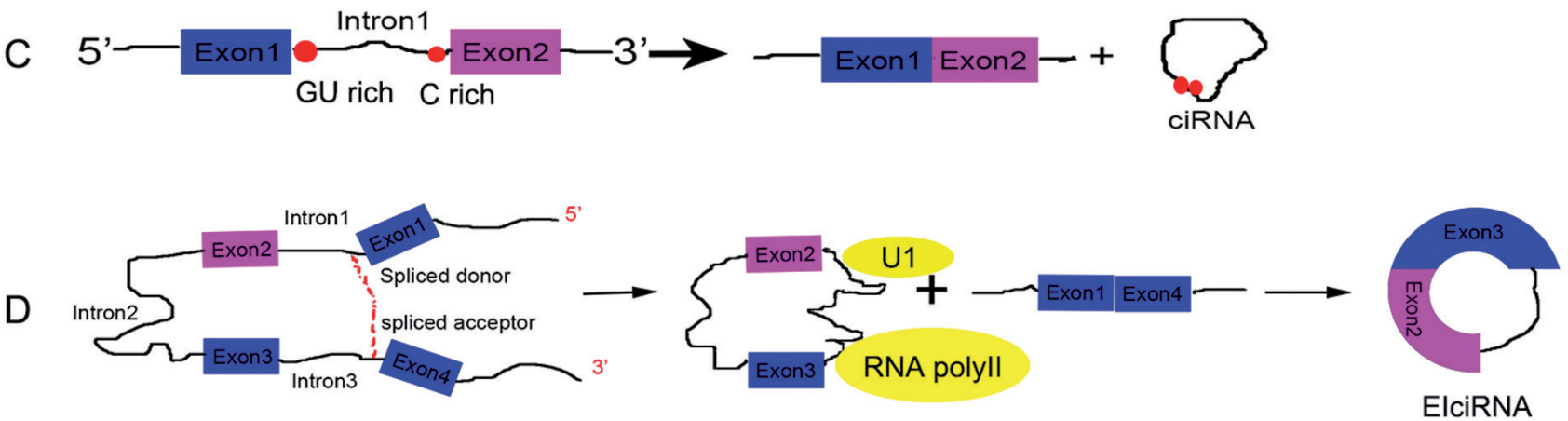

Figure 2. Possible models of circRNAs formation. (A). Lariat-driven circu-larization. Splice donor in 3' end of exon 1 and splice acceptor in 5' end of exon 4 are covalently jointed together, then the introns are removed, and finally exonic circRNA is formed. (B). Intron-pairing-driven circularization. By base-pairing, intron 1 and intron 3 are formed circular structure; then introns are removed to form exonic circRNA. (C). Circular intronic RNA. Intron 1 itself circularizes and then forms ciRNA. (D). EIciRNA is generated due to the retention of internal introns.

At present, circRNA molecules can be divided into three categories according to where in the genome they originated and the composition of their sequence: exonic circRNA [1113], circular intronic RNA (ciRNAs) [14] and retained-exon circRNA [15] (Fig 2). Although the processing mechanism are not fully clear, recent research suggests that each of these three types are produced in completely different ways.

Exonic circRNA. In the past, it was widely accepted that most exons in human precursor messenger RNA (pre-mRNA) were spliced into linear RNA molecules, but in recent years, researchers found that exons are also spliced into circRNA. Exonic circRNA originated from exons in the genome, and the composition of their sequence is only exons, without introns. Formation of exonic circRNA was mainly divided into two models: lariat-driven circularization and intronpairing-driven circularization.

In 2013, Jeck WR et al. [13] put forward two models of circRNA coming from exons: lariat-driven circularization and intron-pairing-driven circularization. The first steps in these two models are different: lariat-driven circularization is initiated through covalent bonding between a splice donor and splice acceptor, while intron-pairing-driven circularization is accomplished through a complementary pair bond between two introns. In the next step, for both models, the spliceosome removes residual introns and forms circRNA. Experimental data is still needed to demonstrate which, if either, model is correct, and to discern how production is controlled. Yang et al. [16] used genome-wide analysis and circRNA recapitulation to show that exon cyclization depends on complementary intron sequences on both sides. They also found that competition between RNA molecules in flanking introns and other intron RNA molecules can affect the efficiency of exon cyclization. Selective RNA pairing and competition between RNA molecules result in selective cyclization, so that one gene can produce a variety of circRNA transcriptions. These results support the intron-pairing-driven circularization theory and show that it is the complementary sequence of introns that mediates exon cyclization, whose product is likely to further increase the known complexity of transcriptional regulation in mammal cells. Recently, German researchers [17] identified 


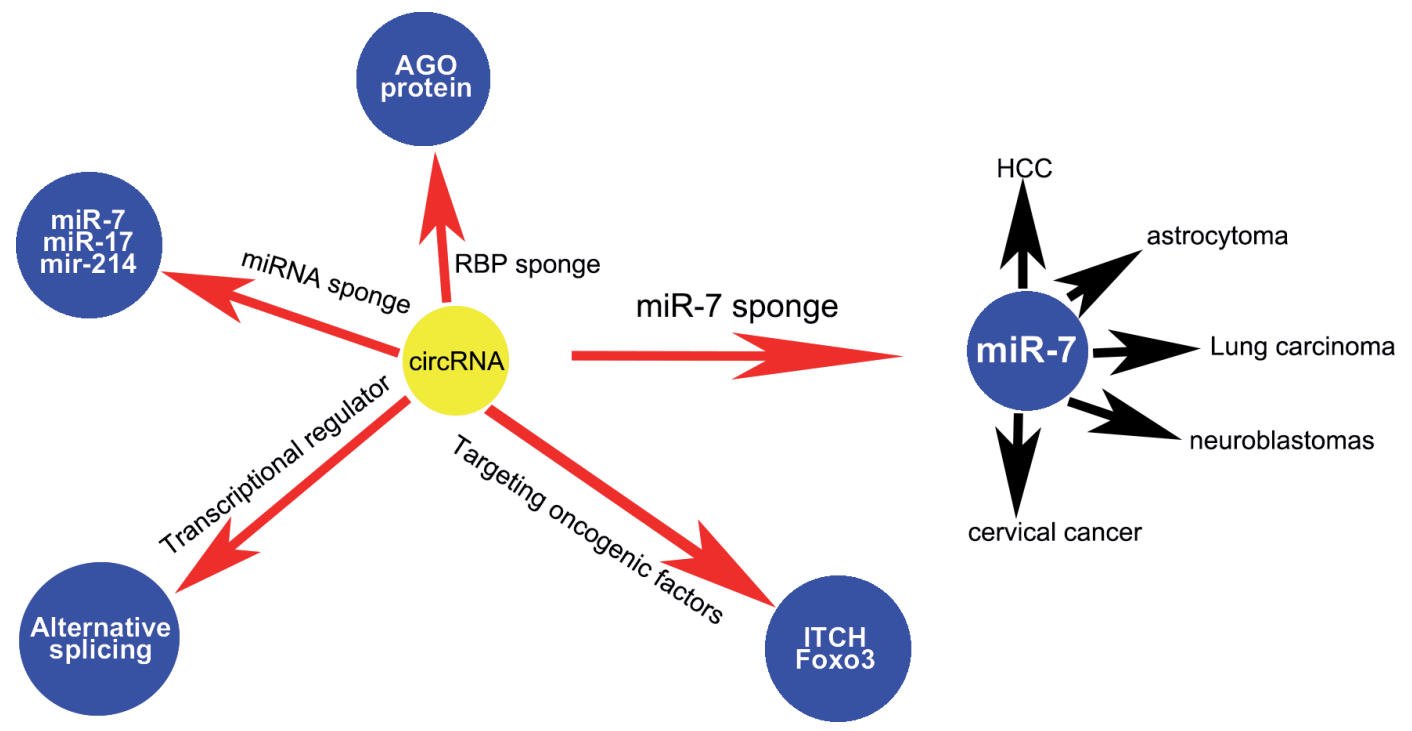

Figure 3. Regulatory network of circRNAs in cancer

circRNA in C. elegans, and reported that introns around the circRNA were highly enriched in the event of RNA editing or super editing. Expression of circRNA can be significantly increased by knocking out the double-stranded RNA-editing deaminase ADAR21. The study provided a model of how animal circRNA forms a larger structure through interaction between RNA molecules. This structure promotes the cyclization of embedded exons.

CircRNA in introns. Although most circRNAs were identified from exons, circRNAs originated from introns emerged in recent research. Most introns form a lasso structure after splicing, and the products are degraded after debranching. The formation of ciRNAs is dependent on the conserved motifs at both ends of the intron, which are the GU-rich motif contained seven nucleotides at the 5 ' splice site and the C-rich motif contained eleven nucleotides at the 3 ' splice site. These motifs inhibit the effect on debranching, thus introns are present in the ring structure. The biggest difference between ciRNAs and exonic circRNAs is that the former is a 2' -5 ' phospholipid-linked cyclized molecule, while the latter is a 3'-5' phospholipid-linked cyclized molecule.

Chen et al. [14] have reported that some introns can form circRNA instead of being debranched after splicing if they contain key sequences. The study also found one kind of ciRNA and verified that it existed stably in a variety of important cells. The ciRNAs were different from known circRNAs in the cytoplasm in their source, generation, regulation, and location, and they were from an intron sequence and regulated by specific cyclization sequences. Mature ciRNAs are located in the position near the transcription locus and regulate transcription of a standard gene through the interaction between ciRNAs and the RNA polymerase II complex. In summary, the circRNA molecules found in the study comprise a new type of long noncoding RNA molecules in terms of structure and concept, and they shed light on the diversity of expression regulation in eukaryotic cells.

Exon-intron circRNA (EIciRNAs). In the process of reverse splicing to form exonic circRNAs, some circular RNA molecules containing un-spliced introns can also be stably present for some reason, these circRNAs are named exon-intron circular RNAs (EIciRNAs). EIciRNAs may be intermediates in the splicing process, or a class of independent circular RNA molecules. Shan Ge et al. [15] identified a class of circRNA related to RNA polymerase II EIciRNAs. RNA polymerase II may catalyze the transcription and synthesis of mRNA and precursors of small nuclear RNA (snRNA) and microRNA. In the study, researchers found that EIciRNAs were located in the nucleus and U1 snRNA genes promoted transcription of their parental gene. These results revealed that circRNA plays a previously-unknown role in regulating gene expression in the nucleus.

\section{ciRS-7/ miR-7 axis on the development of cancer}

circRNAs and cancer. Considering the large a number of circRNAs that have been identified, it is possible that many circRNAs may act as microRNA sponges in regulating the proliferation, invasion and metastasis of cancer. Recent research has indicated that circRNAs may play a crucial role in the initiation and development of some cancers such as breast cancer [18], pancreatic ductal adenocarcinoma [19], oral squamous cell carcinoma [20], gastric carcinoma [21], colorectal cancer [22], esophageal squamous cell carcinoma (ESCC) [23], hepatocellular carcinoma (HCC) $[4,24]$ and many others. In addition, circRNAs are involved in many cancer-related signaling pathways via directly targeting oncogenic factors including mcm5 [20] and Foxo3 [25] (Fig 3). 
Researchers at the University of South Australia found a circRNA-related RBP through high-throughput screening in an RBP siRNA library, providing a preliminary probe for the role of circRNAs in the process of epithelial-mesenchymal transition (EMT). The researchers hope to learn more about circRNA function and develop effective cancer treatments based on these stable transcripts [26]. Chinese scholars [23] have studied changes in circRNA expression in the development of ESCC. The researchers used the Taqman reverse transcription PCR technique to analyze 684 patients to study the relationship between cir-ITCH and ESCC. cir-ITCH is a circular RNA that spans several exons of E3 ubiquitin protein ligases $(I T C H)$. They found that cir-ITCH expression is lower in ESCC compared with adjacent tissue, and verified the function of cir-ITCH through biochemical experiments. As a sponge of miR-7, miR-17, and miR-214, cir-ITCH may increase the ITCH level, which in turn promotes degradation of ubiquitin and phosphorylation of Dvlz, which may inhibit the Wnt/ $\beta$-catenin pathway. Zhu [4] reported knock down of Cdrlas suppressed the HCC cell proliferation and invasion through targeting miR-7. These data suggested that Cdrlas acted as an oncogene partly through targeting miR-7 in HCC. Analogously, Foxo3 circular RNA (circ-Foxo3) can also act as a sponge of potential miRNAs increasing Foxo3 translation thereby suppressing tumor growth, cancer cell proliferation and survival [25]. The circRNAs that act as miRNA sponges are providing us a novel perspective to treat cancer, so more circRNA-miRNA-gene regulatory networks in cancer initiation and progression are needed for further investigations.

miR-7 and cancer. miR-7 can serve as a tumor suppressor that is down-regulated in a variety of cancers, such as hepatocellular carcinoma (HCC) [27], lung neoplasm [28], cervical cancer [29], gastric carcinoma (GC) [30], tongue cancer [31], breast cancer [32] and Schwannoma tumor [33]. It has conclusively been demonstrated that miR-7 is involved in many cancer-related signaling pathways via directly downregulating expression of crucial oncogenic factors including EGF receptor, IRS-1 and IRS-2 [34], Raf1 [35], Ack1 [33], Pak1[36], PIK3CD and mTOR [27].

miR-7 is involved in multiple biological processes of various cancers; for example, it promotes the growth of cervical cancer cells. miR-7 is involved in several pathways related to liver cancer progression, such as the PIK3CD/Akt/mTOR signaling pathway, CCNE1-induced inhibition of cell growth, and the HBx-miR-7-EGFR signaling pathway [27, 37, 38]. Fang [27] confirms that miR-7 expression is significantly lower in HCC cells than in normal cells, suggesting that up-regulation of miR-7 could inhibit the cancer's progression. miR-7 has paired base pairs with PIK3CD, mTOR, and p70S6K, and could effectively regulate the PIK3CD/ Akt/ mTOR signaling pathway by directly targeting these three molecules. The $\mathrm{PIK} 3 \mathrm{CD} / \mathrm{Akt} / \mathrm{mTOR}$ signaling pathway may regulate multiple steps in HCC progression, so that miR-7 would play the role of inhibiting tumor formation and reversing metastasis. Zhang et al. [37] found miR-7 induces growth in G1 of HCC cells, and confirmed CCNE1 is a target of miR-7, so miR-7 could down-regulate CCNE1 to inhibit HCC cell growth. Chen et al. [38] reported that $\mathrm{HBx}$ up regulates miR-7 expression to target the 3'UTR of EGFR mRNA, which in turn reduces EGFR protein expression in HCC cells. HBx-mediated EGFR suppression shifts HCC cells to slow-growth behavior. In contrast, reduction of HBx or miR-7 expression or restoration of EGFR expression can increase the growth rate of HCC cells. Chen demonstrated miR-7-dependent EGFR suppression by $\mathrm{HBx}$, supporting an inhibitory role for HBx in HCC cell growth. These findings not only identify miR-7 as a novel regulatory target of HBx, but also suggest HBx-miR-7-EGFR is a critical signaling pathway in controlling the growth rate of HCC cells.

ciRS-7/miR-7 axis and cancer. It has been implied that cirs-7 likely serves as a crucial factor that is significantly involved in various cancers. miR-7/ciRS-7/target gene regulatory network constructed by CircNet database involved in a variety of cancers, such as Breast cancer [32], Cervical cancer [29], Schwannoma tumor [33], Tongue cancer [31], Lung neoplasm [28], Gastric carcinoma [30], Hepatocellular carcinoma [27]. One study found clinical implication and biological relevance of ciRS-7 in the development and progression of HCC [24]. Moreover, recent research reported knockdown of ciRS-7 suppressed the HCC cell proliferation and invasion through targeting miR-7 in vitro.

Zhu et al. [4] reported ciRS-7 expression was upregulated in HCC tissues compared with the adjacent non-tumor tissues. In addtion, miR-7 expression was downregulated in HCC tissues compared with the adjacent non-tumor tissues. Moreover, the expression level of miR-7 was inversely correlated with ciRS-7 in HCC tissues. Knock down of ciRS-7 suppressed the HCC cell proliferation and invasion through targeting miR-7. These data suggested that ciRS-7 acted as a promoter of the development of HCC partly through targeting miR-7. In addition, a recent review reported that expression analyses of various tumor cell lines showing wide spread expression of ciRS-7 in lung carcinomas, renal cell, neuroblastomas and frequent expression in astrocytoma [39]. There is a large degree of subcellular co-localization between ciRS-7 and miR-7 in HeLa cells when analyzed by IF-fluorescence in situ hybridization and RNA-fluorescence in situ hybridization [2]. The stable expression of ciRS-7 in HeLa cells $[2,11]$ indicates that ciRS-7 may be associated with cervical cancer.

Moreover, ciRS-7 can be used as an ideal ceRNA of miR-7, as it sequesters and potently quenches the normal activities of miR-7 [3]. Furthermore, ciRS-7 is sensitive to miR-671. miR671 can induce the degradation and endonucleolytic cleavage of ciRS-7, so ciRS-7 may be responsible for bringing miR-7 to a subcellular location where miR-671 promotes the release of miR-7 by ciRS-7 [40]. Thus suggesting that miR-671 may inhibit the expression of miR-7. From the interaction of ciRS-7 with cancer associated miR-7, we surmise that they have a potential role in the regulation of cancer (such as cervical cancer).

In summary, miR-7 is closely coupled to ciRS-7 through multiple miRNA response elements (MREs) in the circular 
RNA. It has been implied that fine-tuning of the miR-7/miR$671 /$ ciRS-7 axis will likely serve as profound factors involved in cancer-associated biological processes, which may either promote cancer progression or suppress carcinogenesis depending on the expression level of miRNA target genes.

\section{Detection methods for circRNA}

In the past, sequencing technologies could not identify circular molecules without a 5' terminal cap and 3' poly(A) tail, and early RNA-seq technology could not distinguish them well because of the particular way in which they are produced. For example, exonic circRNAs are produced through back-splice, and early genetic algorithms directly excluded them [1]. But with recent breakthroughs in research techniques, particularly high-throughput sequencing technologies, researchers discovered the existence of circRNA molecules in various species $[11,12]$. As techniques progressed and combinations of different methods improved the efficiency and accuracy of circRNA detection, a solid foundation was laid for further exploring the mechanisms and biological functions of this class of molecules. At present, detection methods for circRNA are as follows:

Molecular biology method. This new technique greatly improves the efficiency of detecting the special structure of these molecules. Separation of poly(A)-RNA and removal of ribosomal RNA (rRNA) was first reported and used in highthroughput sequencing by Yang in 2011 [16]. They focused on poly(A)-RNAs ignored by traditional high-throughput sequencing and reduced the signal interference of poly $(\mathrm{A})+\mathrm{RNA}$. In addition, they discovered that some exons did not belong to the class of poly(A)-RNAs called excised exons; these proved a few years later to be circRNA molecules [12].

Enzymatic methods are also helpful in the detection of circRNA. RNase R, for example, has a strong RNA exonuclease activity from 3' to 5' which can remove linear RNAs or Y-shaped RNAs to collect circRNA molecules specifically [41]. But the exonic circRNA and ciRNAs can resist digestion by RNase R. In addition, some researchers report that tobacco acid pyrophosphatase and terminator 5'-dependent exonuclease can collect circRNA. These two enzymes have the ability to remove the 5 ' terminal cap and cut the fragment from 5' to 3', which can also be used to verify the existence of the circRNA molecules [2].

Danan $\mathrm{M}$ et al. [12] discovered that circRNA moves more slowly in gel than do linear RNAs of the same length because circRNA had no 3' terminal end, and its migration cannot be enhanced by increased gel cross-linking. Based on this difference, they identify circRNA through the northern blot method. Recent studies also suggest that two-dimensional gel electrophoresis and gel trap electrophoresis can confirm RNA cyclization $[1,11]$.

Third, debranching enzymes can distinguish between lariat RNA and circRNA. Both are not sensitive to exonuclease [16], move more slowly than linear RNA [12], and have a ring struc- ture, but circRNA has a backsplice sequence and is not affected by debranching enzymes, while lariat RNAs are.

Genomic method. Several major improvements have been made in the strategy and algorithms of sequencing analysis: (1) It is assumed that there are different forms of exon rearrangements among genes, so it is possible to build a sequence with candidate boundary combinations which may form circRNA, then compare the data with the sequencing [12]. (2) Researchers can match the sequencing data directly with the genome sequence using different sequence alignment algorithms [13]. (3) Researchers can infer the existence of circRNA molecules directly from the cDNA by designing multiple splice joint sequences [42]. Current algorithms applied in the study of circRNA molecules are MapSplice [43], CircSeq [13], and segemehl [44]. Through these methods, researchers have predicted more than 20,000 circRNA molecules, but their accuracy needs further validation [13]. The current genome method has two approaches. One is to select candidate junctions from existing transcript models, which analyze the independent genetic map from paired-end reads sequences at the other end of single cDNA fragments [2]. The other is to match with the reading frame of the genome sequence to identify the joint [45]. In addition, Danan et al. [12] and Jeck et al. [13] use RNase R to digest first, and then analyze undigested RNA using high-throughput sequencing. This technique is called CircleSeq and can detect circRNA molecules; its drawbacks are that it requires a large quantity of RNA and is extremely sensitive to endonuclease contamination.

Others. Recently, researchers established a dedicated database called "circBase," which includes both published circRNA data and the newest circRNA sequencing data [46,47]. Arraystar has taken a lead in developing the first commercial circRNA chips in the world, establishing an experimental platform to study expression of circRNA under different physiological and pathological conditions. Nevertheless, there is still a need for improvement in circRNA research methods.

\section{Perspective}

ciRS-7's involvement in midbrain growth and HCC has been confirmed, but whether it plays a regulating role in other cancers is still unknown. As a sponge of miR-7, ciRS7 down-regulates miR-7 expression by directly binding to miR-7 and inhibiting its activity. miR-7 acts as an important tumor suppressor that inhibits cancer progression at multiple stages, inhibiting tumor formation and reversing metastasis. So ciRS-7/miR-7 axis is postulated to regulate cancer growth and metastasis and ciRS-7 is likely to become a clinical biomarker for cancer diagnosis and therapy. With the advances in a new generation of high-throughput sequencing technologies and other improvements in biological methods, humans have gained deeper understanding of circRNAs molecules from a variety of sources. Of course, much remains to be learned about circRNAs. Research on these and other types 
of RNA molecules will not only enrich our understanding of eukaryotic transcriptomes and the complexity of non-coding RNA, but also provide new ideas and methods for diagnosing and treating cancer and other diseases. Modifying important cancer-related circRNAs binding sites and developing drugs targeting specific circRNAs could potentially change expression levels of downstream target genes and effectively treat cancer.

Acknowledgments: This study was supported by the grants from the National Natural Science Foundation of China (No. 71673193), and the Key Technology Research and Development Program of Sichuan Province (2015SZ0131).

\section{References}

[1] JECK WR, SHARPLESS NE. Detecting and characterizing circular RNAs. Nat Biotechnol 2014; 32: 453-461. https://doi. org/10.1038/nbt.2890

[2] HANSEN TB, JENSEN TI, CLAUSEN BH, BRAMSEN JB, FINSEN B et al. Natural RNA circles function as efficient microRNA sponges. Nature 2013; 495: 384-388. https://doi. org/10.1038/nature11993

[3] MEMCZAK S, JENS M, ELEFSINIOTI A, TORTI F, KRUEGER J et al. Circular RNAs are a large class of animal RNAs with regulatory potency. Nature 2013; 495: 333-338. https:// doi.org/10.1038/nature11928

[4] YU L, GONG X, SUN L, ZHOU Q, LU B et al. The Circular RNA Cdrlas Act as an Oncogene in Hepatocellular Carcinoma through Targeting miR-7 Expression. PLoS One 2016; 11: e0158347. https://doi.org/10.1371/journal.pone.0158347

[5] FERLAY J, SOERJOMATARAM I, DIKSHIT R, ESER $S$, MATHERS $\mathrm{C}$ et al. Cancer incidence and mortality worldwide: sources, methods and major patterns in GLOBOCAN 2012. Int J Cancer 2015; 136: E359-386. https://doi. org/10.1002/ijc.29210

[6] BLACK DL. Mechanisms of alternative pre-messenger RNA splicing. Annu Rev Biochem 2003; 72: 291-336. https://doi. org/10.1146/annurev.biochem.72.121801.161720

[7] CAPEL B, SWAIN A, NICOLIS S, HACKER A, WALTER M et al. Circular transcripts of the testis-determining gene Sry in adult mouse testis. Cell 1993; 73: 1019-1030. https://doi. org/10.1016/0092-8674(93)90279-Y

[8] COCQUERELLE C, DAUBERSIES P, MAJERUS MA, KERCKAERT JP, BAILLEUL B. Splicing with inverted order of exons occurs proximal to large introns. EMBO J 1992; 11: 1095-1098.

[9] NIGRO JM, CHO KR, FEARON ER, KERN SE, RUPPERT JM et al. Scrambled exons. Cell 1991; 64: 607-613. https://doi. org/10.1016/0092-8674(91)90244-S

[10] COCQUERELLE C, MASCREZ B, HETUIN D, BAILLEUL B. Mis-splicing yields circular RNA molecules. FASEB J 1993; 7: 155-160.

[11] SALZMAN J, GAWAD C, WANG PL, LACAYO N, BROWN PO. Circular RNAs are the predominant transcript isoform from hundreds of human genes in diverse cell types. PLoS One 2012; 7: e30733. https://doi.org/10.1371/journal.pone.0030733
[12] DANAN M, SCHWARTZ S, EDELHEIT S, SOREK R. Transcriptome-wide discovery of circular RNAs in Archaea. Nucleic Acids Res 2012; 40: 3131-3142. https://doi. org/10.1093/nar/gkr1009

[13] JECK WR, SORRENTINO JA, WANG K, SLEVIN MK, BURD $\mathrm{CE}$ et al. Circular RNAs are abundant, conserved, and associated with ALU repeats. RNA 2013; 19: 141-157. https://doi. org/10.1261/rna.035667.112

[14] ZHANG Y, ZHANG XO, CHEN T, XIANG JF, YIN QF et al. Circular intronic long noncoding RNAs. Mol Cell 2013; 51: 792-806. https://doi.org/10.1016/j.molcel.2013.08.017

[15] LI Z, HUANG C, BAO C, CHEN L, LIN M et al. Exon-intron circular RNAs regulate transcription in the nucleus. Nat Struct Mol Biol 2015; 22: 256-264. https://doi.org/10.1038/ nsmb.2959

[16] YANG L, DUFF MO, GRAVELEY BR, CARMICHAEL GG, CHEN LL. Genomewide characterization of nonpolyadenylated RNAs. Genome Biol 2011; 12: R16. https:// doi.org/10.1186/gb-2011-12-2-r16

[17] RYBAK-WOLF A, STOTTMEISTER C, GLAZAR P, JENS M, PINO $\mathrm{N}$ et al. Circular RNAs in the Mammalian Brain Are Highly Abundant, Conserved, and Dynamically Expressed. Mol Cell 2015; 58: 870-885. https://doi.org/10.1016/j.molcel.2015.03.027

[18] ZHANG C, WU H, WANG Y, ZHAO Y, FANG X et al. Expression Patterns of Circular RNAs from Primary Kinase Transcripts in the Mammary Glands of Lactating Rats. J Breast Cancer 2015; 18: 235-241. https://doi.org/10.4048/ jbc.2015.18.3.235

[19] MCGLYNN LM, MCCLUNEY S, JAMIESON NB, THOMSON J, MACDONALD AI et al. SIRT3 \& SIRT7: Potential Novel Biomarkers for Determining Outcome in Pancreatic Cancer Patients. PLoS One 2015; 10: e0131344. https://doi. org/10.1371/journal.pone.0131344

[20] YU SY, WANG YP, CHANG JY, SHEN WR, CHEN HM et al. Increased expression of MCM5 is significantly associated with aggressive progression and poor prognosis of oral squamous cell carcinoma. J Oral Pathol Med 2014; 43: 344-349. https:// doi.org/10.1111/jop.12134

[21] LI P, CHEN S, CHEN H, MO X, LI T et al. Using circular RNA as a novel type of biomarker in the screening of gastric cancer. Clin Chim Acta 2015; 444: 132-136. https://doi.org/10.1016/j. cca.2015.02.018

[22] DE WIT M, KANT H, PIERSMA SR, PHAM TV, MONGERA $S$ et al. Colorectal cancer candidate biomarkers identified by tissue secretome proteome profiling. J Proteomics 2014; 99: 26-39. https://doi.org/10.1016/j.jprot.2014.01.001

[23] LI F, ZHANG L, LI W, DENG J, ZHENG J et al. Circular RNA ITCH has inhibitory effect on ESCC by suppressing the Wnt/ beta-catenin pathway. Oncotarget 2015; 6: 6001-6013. https:// doi.org/10.18632/oncotarget.3469

[24] XU L, ZHANG M, ZHENG X, YI P, LAN C et al. The circular RNA ciRS-7 (Cdrlas) acts as a risk factor of hepatic microvascular invasion in hepatocellular carcinoma. J Cancer Res Clin Oncol 2016; Epub ahead of print.

[25] YANG W, DU WW, LI X, YEE AJ, YANG BB. Foxo3 activity promoted by non-coding effects of circular RNA and Foxo3 
pseudogene in the inhibition of tumor growth and angiogenesis. Oncogene 2016; 35: 3919-3931. https://doi.org/10.1038/ onc. 2015.460

[26] CONN SJ, PILLMAN KA, TOUBIA J, CONN VM, SALMANIDIS $\mathrm{M}$ et al. The RNA binding protein quaking regulates formation of circRNAs. Cell 2015; 160: 1125-1134. https:// doi.org/10.1016/j.cell.2015.02.014

[27] FANG Y, XUE JL, SHEN Q, CHEN J, TIAN L. MicroRNA-7 inhibits tumor growth and metastasis by targeting the phosphoinositide 3-kinase/Akt pathway in hepatocellular carcinoma. Hepatology 2012; 55: 1852-1862. https://doi.org/10.1002/ hep. 25576

[28] LI J, ZHENG Y, SUN G, XIONG S. Restoration of miR-7 expression suppresses the growth of Lewis lung cancer cells by modulating epidermal growth factor receptor signaling. Oncol Rep 2014; 32: 2511-2516. https://doi.org/10.3892/ or.2014.3519

[29] LIU S, ZHANG P, CHEN Z, LIU M, LI X et al. MicroRNA-7 downregulates XIAP expression to suppress cell growth and promote apoptosis in cervical cancer cells. FEBS Lett 2013; 587: 2247-2253. https://doi.org/10.1016/j.febslet.2013.05.054

[30] KONG D, PIAO YS, YAMASHITA S, OSHIMA H, OGUMA Ket al. Inflammation-induced repression of tumor suppressor miR-7 in gastric tumor cells. Oncogene 2012; 31: 3949-3960. https://doi.org/10.1038/onc.2011.558

[31] JIANG L, LIU X, CHEN Z, JIN Y, HEIDBREDER CE et al. MicroRNA-7 targets IGF1R (insulin-like growth factor 1 receptor) in tongue squamous cell carcinoma cells. Biochem J 2010; 432: 199-205. https://doi.org/10.1042/BJ20100859

[32] ZHANG H, CAI K, WANG J, WANG X, CHENG $\mathrm{K}$ et al. MiR-7, inhibited indirectly by lincRNA HOTAIR, directly inhibits SETDB1 and reverses the EMT of breast cancer stem cells by downregulating the STAT3 pathway. Stem Cells 2014; 32: 2858-2868. https://doi.org/10.1002/stem.1795

[33] SAYDAM O, SENOL O, WURDINGER T, MIZRAK A, OZDENER GB et al. miRNA-7 attenuation in Schwannoma tumors stimulates growth by upregulating three oncogenic signaling pathways. Cancer Res 2011; 71: 852-861. https:// doi.org/10.1158/0008-5472.CAN-10-1219

[34] KEFAS B, GODLEWSKI J, COMEAU L, LI Y, ABOUNADER $\mathrm{R}$ et al. microRNA-7 inhibits the epidermal growth factor receptor and the Akt pathway and is down-regulated in glioblastoma. Cancer Res 2008; 68: 3566-3572. https://doi. org/10.1158/0008-5472.CAN-07-6639

[35] WEBSTER RJ, GILES KM, PRICE KJ, ZHANG PM, MATTICK JS et al. Regulation of epidermal growth factor receptor signaling in human cancer cells by microRNA-7. J Biol Chem 2009; 284: 5731-5741. https://doi.org/10.1074/jbc. M804280200
[36] REDDY SD, OHSHIRO K, RAYALA SK, KUMAR R. MicroRNA-7, a homeobox D10 target, inhibits p21-activated kinase 1 and regulates its functions. Cancer Res 2008; 68: 8195-8200. https://doi.org/10.1158/0008-5472.CAN-08-2103

[37] ZHANG X, HU S, ZHANG X, WANG L, ZHANG X et al. MicroRNA-7 arrests cell cycle in G1 phase by directly targeting CCNE1 in human hepatocellular carcinoma cells. Biochem Biophys Res Commun 2014; 443: 1078-1084. https://doi. org/10.1016/j.bbrc.2013.12.095

[38] CHEN YJ, CHIEN PH, CHEN WS, CHIEN YF, HSU YY et al. Hepatitis B Virus-Encoded X Protein Downregulates EGFR Expression via Inducing MicroRNA-7 in Hepatocellular Carcinoma Cells. Evid Based Complement Alternat Med 2013; 2013: 682380. https://doi.org/10.1155/2013/682380

[39] HANSEN TB, KJEMS J, DAMGAARD CK. Circular RNA and miR-7 in cancer. Cancer Res 2013; 73: 5609-5612. https://doi. org/10.1158/0008-5472.CAN-13-1568

[40] KOSIK KS Molecular biology: Circles reshape the RNA world. Nature 2013; 495: 322-324.

[41] SUZUKI H, ZUO Y, WANG J, ZHANG MQ, MALHOTRA A et al. Characterization of RNase R-digested cellular RNA source that consists of lariat and circular RNAs from pre-mRNA splicing. Nucleic Acids Res 2006; 34: e63. https:// doi.org/10.1093/nar/gkl151

[42] HOFFMANN S, OTTO C, DOOSE G, TANZER A, LANGENBERGER D et al. A multi-split mapping algorithm for circular RNA, splicing, trans-splicing and fusion detection. Genome Biol 2014; 15: R34. https://doi.org/10.1186/gb-2014$\underline{15-2-r 34}$

[43] WANG K, SINGH D, ZENG Z, COLEMAN SJ, HUANG Y et al. MapSplice: accurate mapping of RNA-seq reads for splice junction discovery. Nucleic Acids Res 2010; 38: e178. https:// doi.org/10.1093/nar/gkq622

[44] SALZMAN J, CHEN RE, OLSEN MN, WANG PL, BROWN PO. Cell-type specific features of circular RNA expression. PLoS Genet 2013; 9: e1003777. https://doi.org/10.1371/journal.pgen. 1003777

[45] HANSEN TB, WIKLUND ED, BRAMSEN JB, VILLADSEN $\mathrm{SB}$, STATHAM AL et al. miRNA-dependent gene silencing involving Ago2-mediated cleavage of a circular antisense RNA. EMBO J 2011; 30: 4414-4422. https://doi.org/10.1038/ emboj.2011.359

[46] GLAZAR P, PAPAVASILEIOU P, RAJEWSKY N. circBase: a database for circular RNAs. RNA 2014; 20: 1666-1670. https://doi.org/10.1261/rna.043687.113

[47] LIU YC, LI JR, SUN CH, ANDREWS E, CHAO RF et al CircNet: a database of circular RNAs derived from transcriptome sequencing data. Nucleic Acids Res 2016; 44: D209-215. https://doi.org/10.1093/nar/gkv940 